\title{
Cannabis medicinal en Argentina: perspectiva desde la salud pública
}

\author{
Medicinal cannabis in Argentina: a public health perspective
}

Ornella Agnese $^{\mathrm{a}}$, Rocio Macarena Sueiro López ${ }^{\mathrm{a}}$, lara Yamila Taito Vicenti ${ }^{\mathrm{a}}$, Juan Víctor Ariel Franco ${ }^{\mathrm{a}, \mathrm{b}}$

\begin{abstract}
Resumen
Esta revisión analiza la situación actual de la utilización del cannabis como herramienta terapéutica dentro del ámbito de la salud en Argentina, haciendo referencia a los distintos actores involucrados y dilemas futuros que pueden presentarse. Para comprender en su totalidad el marco social, cultural e histórico, se desarrollan distintos aspectos, como la descripción química y biológica del cannabis, evolución del consumo a través de la historia, las repercusiones del consumo recreacional y las distintas aplicaciones que tiene en el campo de la medicina. También se describen las diferentes realidades que hay en el mundo, así como las legislaciones de otros países y la comparación de estas con la que tenemos en nuestro país. Finalmente se mencionan los desafíos pendientes y sus posibles abordajes.

Abstract

This review analyzes the current situation of the use of cannabis as a therapeutic tool in the field of health in Argentina, referring to the different actors involved and future dilemmas that may arise. To fully understand the social, cultural and historical framework, different aspects can be defined, such as the chemical and biological description of cannabis, the evolution of consumption throughout history, the repercussions of recreational consumption and the different applications that it has on the medical field. It also describes the different realities that exist in the world, as well as the laws of other countries and the comparison of these with the one we have in our country. Finally, the pending challenges and their possible approaches, are mentioned.
\end{abstract}

Palabras clave: Cannabis, Terapias Complementarias. Keywords: Cannabis, complementary therapies.

Agnese O, Sueiro López RM, Taito Vicenti IY, Franco JVA.. Cannabis medicinal en Argentina: perspectiva desde la salud pública. Evid Actual Pract Ambul. 2019;22(1):e001119.

\section{Introducción}

La planta de Cannabis es una planta dioica, con flores macho y hembra que usualmente crecen en plantas separadas, con un desarrollo anual. Se identifican dos subespecies principales: Cannabis sativa, de finas hojas verdes claras, y Cannabis indica, de menor altura y hojas más oscuras por su menor contenido de tetrahidrocannabinol. Se cree que su origen se encuentra en Asia central, siendo su primera plantación en China y luego en India. Durante la historia esta planta fue utilizada con diversos propósitos como la obtención de fibra, el uso de sus semillas como alimento, el alivio de distintas enfermedades o síntomas, y los conocidos efectos psicoactivos. Actualmente, el cannabis es la droga ilegal más utilizada en el mundo ${ }^{1}$.

De los más de 500 componentes presentes en la planta de cannabis, por lo menos 113 de ellos corresponden a cannabinoides, más específicamente fitocannabinoides, un tipo de químico capaz de interactuar con los receptores de cannabinoides celulares que alteran la liberación de ciertos neurotransmisores en el sistema nervioso. Dentro de estos los más destacados se encuentran el delta-9-tetrahidrocannabinol (THC), principal componente psicoactivo de la planta, y el cannabidiol (CBD). EI THC actúa como agonista parcial de la actividad de los receptores de cannabinoides $\mathrm{CB} 1$ y $\mathrm{CB} 2$ reduciendo los niveles de AMPc, mientras que el CBD tiene baja afinidad por dichos receptores, aunque actúa como un antagonista indirecto. Aunque ambos componentes parecieran tener efectos similares sobre estos dominios, tienen efectos opuestos en otros aspectos, como por ejemplo en los efectos psicotrópicos y antipsicóticos ${ }^{1-3}$.

En la actualidad el dilema sobre su uso se encuentra en la aplicación de la misma dentro del ámbito médico para el alivio de distintas enfermedades o síntomas, lo que se conoce como cannabis o marihuana medicinal.

\section{Historia del uso del cannabis}

El uso del cannabis comenzó en conjunto con el descubrimiento de la agricultura. Si bien en un principio era utilizada por su fibra, uno de los primeros datos de su uso medicinal fue aproximadamente en 2700 a.C. como una medicina para el tratamiento del reuma, la gripe y el paludismo ${ }^{4}$. En Europa continental se introdujo su uso medicinal en el siglo XIII como anticonvulsivante, analgésico, ansiolítico y antiemético, y se registraron los primeros casos de su uso como estimulante. En Gran Bretaña, el divulgador de su aplicación terapéutica fue O'Shaughnessy, quien había descubierto su uso al trabajar con el ejército en India. En Francia, al contrario, se utilizaba por sus efectos psicoactivos que generaron una gran influencia literaria ${ }^{5}$. Por su parte, en la América colonial, el cannabis fue introducido para la obtención de fibra en los Virreinatos de Perú y de México por los conquistadores españoles, y en Canadá y Estados Unidos por los colonos franceses e ingleses. En el siglo XVII comenzó a ser fumado por esclavos, costumbre que se extendió durante el siglo XIX a las poblaciones agrarias de Centroamérica y México, y de allí, a través de migraciones a Texas y Luisiana, Estados Unidos ${ }^{5}$

El primer compuesto aislado de Cannabis sativa fue el cannabinol (CBN) en 1899, pero fue caracterizado en 1963 y se descubrió que no era responsable principal de los efectos alucinógenos del cannabis ${ }^{5}$. Otro de sus componentes activos, el $\Delta 9$ tetrahidrocannabinol ( $\Delta 9-\mathrm{THC})$ fue aislado en 1964 y permitió la investigación científica de sus propiedades biológicas. Se logró el desarrollo de un derivado con capacidad terapéutica al conseguir separar las propiedades farmacológicas de los efectos psicoacti-

\footnotetext{
a Instituto Universitario Hospital Italiano. iara.taito@hospitalitaliano.org.ar

b Servicio de Medicina Familiar y Comunitaria, Hospital Italiano de Buenos Aires. juan.franco@hospitalitaliano.org.ar
} 
$\operatorname{vos}^{4}$

La primera restricción federal para el uso y venta de cannabis en Estados Unidos fue en el año 1937 con la generación del impuesto a la marihuana. Como acto subsecuente, en 1942 se retira el cannabis de las farmacias de este país. Su tenencia y consumo fue creciendo en cargos hasta llegar a la prohibición total bajo la ley federal en $1970^{6}$

\section{Consecuencias negativas del consumo de cannabis}

Las consecuencias negativas del consumo de cannabis como droga recreacional es uno de los factores que influyen en la opinión desfavorable de las personas al momento de discutir su implementación dentro de la medicina.

A nivel cerebral está correlacionado con modificaciones anatómicas, como adelgazamiento de la corteza o daños sobre el hipocampo $^{7,8}$, lo que se podría asociar a problemas de memoria e impulsividad ${ }^{9}$. Como síntomas neurológicos asociados al consumo los que más se destacan son: alucinaciones, principalmente auditivas, menor calidad del sueño con adormecimiento diurno, delirios, alteración en funciones visual-motoras y percepción y memoria visual de evocación inmediata ${ }^{10,11}$

Con respecto al sistema respiratorio los efectos varían desde episodios de tos en la mañana hasta bronquiolitis y función pulmonar afectada ${ }^{12}$. En comparación con el consumo de tabaco ambas aumentan la prevalencia de enfermedad pulmonar obstructiva crónica por joint year o pack year. Se observó que el consumo conjunto de ambas sustancias genera síntomas más pronunciados $^{13}$. No hay evidencia que relacione el consumo de cannabis con eventos cardiovasculares ${ }^{14}$

En relación al consumo durante el embarazo, la exposición prenatal tendría consecuencias en el desarrollo neuronal ${ }^{15}$ y mayor riesgo de muerte fetal ${ }^{16}$.

En los casos de intoxicaciones agudas por cannabis los efectos adversos más frecuentes son: agitación y agresión, psicosis, ansiedad y vómitos ${ }^{17}$. Es importante destacar que, al igual que sucede con los fumadores pasivos del tabaco, los efectos adversos que pueden desencadenarse por el consumo recreativo de cannabis afectan tanto a aquellos que consumen directamente, como a quienes se encuentran en el mismo ambiente ${ }^{18}$.

Por otro lado, los posibles efectos adversos no se restringen a componentes biológicos, sino que también tiene componentes sociales como pueden ser los problemas académicos o trastornos anímicos en adolescentes que consumen ${ }^{19,20}$. También se lo puede ligar a situaciones de accidentes, como intoxicación de infantes por ingesta accidental, frecuentemente entre los 1,5 a 2 años ${ }^{21}$ o siniestros viales ya que disminuye la habilidad psicomotriz y altera la cognición ${ }^{22}$.

\section{Aplicaciones medicinales del cannabis \\ Dolor y cuidados paliativos}

La utilización de cannabis en el dolor relacionado al cáncer, demostró efecto analgésico comparado con placebo; y en altas dosis se reportaron efectos secundarios como cambios en la cognición, sedación y mareos ${ }^{23}$. También para el tratamiento del dolor no relacionado al cáncer se demostraron efectos analgésicos significativos. Los efectos adversos reportados fatiga y mareo) fueron generalmente bien tolerados ${ }^{24}$. Con respecto al dolor neuropático crónico y al dolor agudo la evidencia sobre la reducción del dolor es de muy baja calidad. Se sostiene que, en comparación, los beneficios potenciales de la medicina basada en cannabis son sobrepasados por su daño potencial ${ }^{25,26}$.

Este es uno de los principales usos del cannabis medicinal: entre el $45 \%$ y el $80 \%$ de las personas buscan cannabis medicinal para manejo del dolor, y el $39 \%$ de ellas están en tratamiento con opioides a largo plazo ${ }^{27}$. Entre aquellos pacientes con dolores crónicos, se estima que la prevalencia del consumo oscila entre el $12 \%$ y el $15 \% 27,28$.

Con respecto al uso de cannabinoides en la medicina paliativa en oncología, no mostró diferencias significativas en comparación con placebo para mejorar la ingesta calórica, apetito, náuseas y vómitos, disminución del dolor y los problemas para dormir. En pacientes con $\mathrm{VIH}$, se comprobó que estimulaba la ganancia de peso, aunque los efectos eran superados por otro fármaco, megestrol (tratamiento hormonal), por lo que no hay evidencia que sugiera que los cannabinoides sean valiosos en ninguna de las dos situaciones ${ }^{29,30}$.

\section{Epilepsia}

En relación con el tratamiento de la epilepsia, se ha comenzado a obtener evidencia científica que sugiere la efectividad del CBD en la reducción y cese de convulsiones en pacientes con síndrome de Dravet y el síndrome de Lennox-Gastaut; ambas encefalopatías epilépticas. Experiencias sobre el uso de CBD apoyan la eficacia de este en un amplio rango de epilepsias resistentes al tratamiento. Con respecto al uso de THC, ya sea solo o en combinación con $\mathrm{CBD}$, todavía no está determinada su efectividad y seguridad en el uso ${ }^{31}$.

Mediante una encuesta telefónica en Canadá, se estimó que el $21 \%$ de los pacientes con epilepsia habían utilizado cannabis en el pasado año y reportaron mejoras en sus episodios epilépticos $^{32}$. En Europa, el cannabidiol es una terapia no aprobada y su uso no es tan frecuente. Aún así, una encuesta pan-Europea evidenció que hasta un $7 \%$ de los niños y adolescentes con sindromes epilépticos han usado cannabinoides ${ }^{33}$.

\section{Otras patologías}

Profundizando en patologías específicas, ni la espasticidad por esclerosis múltiple ni el trastorno de estrés post traumático ${ }^{34}$, síndrome de Tourette ${ }^{35}$ ni trastornos del estado de ánimo presentan beneficios con el tratamiento con cannabis o al menos su uso es incierto todavía. Con respecto a la esclerosis múltiple, una encuesta realizada en Canadá dio como resultado que la mayoría sabia sobre el uso de cannabis para los síntomas asociados con su enfermedad (96\%). Del grupo que había probado cannabis $(n=176), 35 \%$ fue para manejar los síntomas, y entre ellos 43 personas $(64 \%)$ la utilizaban al momento de la encuesta y 24 (36\%) había discontinuado su uso por el costo, los efectos adversos, falta de eficacia o problemas legales ${ }^{36}$. Por otra parte, en una encuesta en el Reino Unido, $43 \%$ de los participantes usaban el cannabis en forma terapéutica para mitigar los espasmos y aliviar el dolor ${ }^{37}$. En Nueva Escocia, de 220 pacientes $14 \%$ usaba regularmente cannabis para alivio de los síntomas, entre ellos estrés, problemas del sueño, espasmos, humor y dolor ${ }^{38}$.

Las aplicaciones realmente efectivas del cannabis medicinal son mucho menores a las generalmente difundidas. Además sus efectos beneficiosos suelen ser sobrepasados por los efectos adversos que genera su consumo, dejando de ser una opción de tratamiento eficaz y segura para ofrecer a los pacientes. Las únicas aplicaciones que parecerían ser efectivas, son el control del dolor no agudo y en el manejo de epilepsias resistentes al tratamiento.

\section{Legislaciones internacionales sobre el cannabis Holanda}

La legislación holandesa proviene de la reforma fundamental realizada sobre la Ley de Opio en el año 1976. Dicha reforma se basó en tres pilares fundamentales: estricta acción contra el narcotráfico, principalmente de las drogas duras, y de los delitos relacionados a las drogas, legalización del consumo personal de drogas blandas y venta de pequeñas cantidades para dicho fin, y asistencia para aquellos que tengan dependencia a las drogas 39 . El consumo de drogas blandas se redujo considerablemente tras su legalización. 
Actualmente es posible adquirir marihuana en Holanda siendo mayor de 18 años hasta un máximo de $5 \mathrm{~g}$. al día. A aquellos turistas que deseen consumir, el acceso a la marihuana varía según cada ayuntamiento, aunque hay sitios donde pueden obtenerla, como, por ejemplo, Amsterdam ${ }^{40}$. En relación con los fines medicinales, Holanda fue el primer país en dispensar marihuana medicinal en las farmacias, tras su aprobación en marzo del $2003^{41}$. La producción con fines medicinales y científicos está a cargo de la Oficina de Marihuana Medicinal, dependiente del gobierno. Esta agencia posee el monopolio del abastecimiento de las farmacias, hospitales y veterinarias con cannabis y resina de cannabis, y de la regulación de la importación y exportación ${ }^{42}$. Según el anexo 1 del informe de cannabinoides, "situación ante las agencias de medicamentos en países de alta vigilancia sanitaria" se menciona que en Países Bajos está aprobado el SATIVEX ${ }^{\circledR}$ (Delta-9-tetrahidrocannabinol/cannabidiol). El mismo se utiliza en casos de espasticidad por esclerosis múltiple ${ }^{43}$.

\section{Uruguay}

El 7 de enero de 2014 se promulgó la ley 19.172 "Marihuana y sus derivados", que trata sobre su importación, producción, adquisición, almacenamiento, comercialización y distribución. El objetivo de esta ley es la reducción de daños por el uso del cannabis (como el comercio ilegal, narcotráfico y crimen organizado), la promoción de información y educación sobre el tema y la reinserción social de los usuarios problemáticos de drogas. El estado uruguayo asumió el control y regulación de la importación, exportación, plantación y distribución; para su comercialización y distribución. En esta ley se incluía también el cultivo de cáñamo (cannabis no psicoactivo) y de marihuana medicinal ${ }^{44}$. A diferencia de la ley argentina que solo trata sobre el cannabis medicinal y para investigación, en esta reforma se incluye la regulación del cannabis psicoactivo (con THC natural mayor a $1 \%$ ), del cáñamo (cannabis no psicoactivo), del cannabis para investigación y el uso farmacéutico, y la plantación doméstica (hasta 480 gramos anuales) y por clubes de membresía (recolección anual proporcional a número de socios) ${ }^{44}$. Para su uso científico o medicinal deberán ser autorizados y controlados directamente por el Ministerio de Salud Pública, a través del Instituto de Regulación y Control de Cannabis (IRCCA). Posteriormente se menciona que a las farmacias se les otorgan licencias de expendio de cannabis psicoactivo, para lo cual las personas requieren una acreditación o una receta médica en caso de marihuana medicinal; y no podrán superar los 40 gramos de marihuana no medicinal mensuales por usuario 44

\section{Estados Unidos}

En el estado de California el uso medicinal del cannabis fue aprobado en el año 1996, siendo el primero en legislar sobre dicho uso ${ }^{45}$. A partir de este hecho, se han sumado 29 de los 50 estados de este país y el distrito de Columbia, Guam y Puerto Rico. Estas legislaciones permiten programas integrales de marihuana medicinal pública y cannabis.

A nivel federal, la marihuana sigue clasificada como tipo I en la Ley de Sustancias Controladas ${ }^{46}$, donde se considera que las sustancias de esta clasificación tienen un alto potencial de dependencia y ningún uso médico aceptado, considerando su distribución como una ofensa federal. Debido a esta clasificación la prescripción del cannabis medicinal es inválida, ya que queda prohibida por la ley federal. Esto lleva a que habitualmente las prescripciones se denominan como "recomendaciones" o "derivaciones". Los estados con legislaciones sobre la aplicación medicinal del cannabis generalmente poseen un registro sobre los pacientes que reciben dicha droga. El mismo puede actuar como herramienta de protección ante posibles problemas legales por la tenencia o consumo.
El instituto de Medicina de Estados Unidos publicó un reporte en el cual establecen un potencial valor terapéutico de las drogas cannabinoides para el manejo del dolor, control de náuseas y vómitos y la estimulación del apetito ${ }^{47}$. También destaca un valor terapéutico potencial sobre los efectos psicológicos como la reducción de la ansiedad, la sedación y la euforia, pero que también dichos efectos pueden dificultar la interpretación de otros aspectos de la funcionalidad de la droga. Además, el Cannabidiol fue regulado por la administración de medicamentos y alimentos de Estados Unidos como una droga huérfana. Esto quiere decir que se trata de medicamentos que no se desarrollan por la industria farmacéutica porque van destinados a un grupo reducidos de pacientes, pero que es relevante para la salud pública 48 Los usos de cannabidiol fueron: tratamiento del síndrome de Dravet, tratamiento de glioblastoma multiforme, síndrome LennoxGastaut, esquizofrenia pediátrica, y glioma, espasmos infantiles, prevención de injerto versus huésped, tratamiento de encefalopatía hipóxica isquémica neonatal; $y$ tratamiento del síndrome del $\mathrm{X}$ frágil 49 .

\section{Legislación Argentina sobre el cannabis medicinal}

El primer antecedente de legislación sobre el cannabis en nuestro país es la Ley 27.737, llamada "Régimen penal de estupefacientes". La misma reguló el uso, cultivo y distribución del cannabis desde el 11 de octubre de 1989 hasta el año 2017. La única mención sobre su uso en la práctica médica, en el artículo 9ㅇ, establece una multa y la consiguiente pena a aquel médico que recete o suministre estupefacientes fuera de tratamiento o por fuera de las cantidades establecidas"; pero dentro de la ley no se menciona ni regula el uso de manera específica ${ }^{50}$.

Con el objetivo de facilitar el acceso al aceite de cannabis medicinal, el 29 de Marzo de 2017 fue sancionada la Ley 27.350 que regula la investigación y el uso medicinal del Cannabis bajo el nombre de "Ley de Uso Medicinal de la Planta de Cannabis" 51,52 El artículo $1^{\circ}$ de la misma plantea que el objeto de la ley es el establecimiento de un marco regulatorio para la investigación médica y científica del uso medicinal, terapéutico y/o paliativo del dolor de la planta de cannabis y sus derivados. Para ello, según el artículo $2^{\circ}$, se crea el Programa Nacional para el Estudio y la Investigación del Uso Medicinal de la Planta de Cannabis, sus derivados y tratamientos no convencionales, dependiente del Ministerio de Salud. Los objetivos de dicho programa están expuestos en el Artículo 3 ‥ Entre ellos destacan la promoción de medidas de concientización, el desarrollo de evidencia científica, y el acceso gratuito al aceite de cáñamo y derivados del cannabis a aquellos que se incorporen al programa y cumplan las condiciones, además de asesoramiento, cobertura adecuada y completo seguimiento del tratamiento. Dentro de la investigación, el foco se establece en los fines terapéuticos y científicos de la planta de cannabis, su eficacia y el impacto en el organismo humano. De esta medida se desprende otro objetivo del programa: "Conocer los efectos secundarios del uso medicinal de la planta de cannabis y sus derivados, y establecer la seguridad y las limitaciones para su uso, promoviendo el cuidado de la población en su conjunto".

Para ello, la regulación de la ley autoriza al Consejo Nacional de Investigación Científica y Técnicas (CONICET), y al Instituto Nacional de Tecnología Agropecuaria (INTA) el cultivo de Cannabis para la investigación médica y la elaboración de sustancia como medicamento. Incluye también la participación voluntaria de pacientes bajo tratamientos y sus familiares, con el fin de nutrirse de su experiencia, conocimiento empírico, vivencias y métodos utilizados para su autocuidado; y de la capacitación continua de profesionales de la salud 52

Según la reglamentación de dicha ley, las personas incluidas bajo este programa son aquellas que padecen "una enfermedad 
bajo parámetros de diagnósticos específicos y clasificados por la Organización Mundial de Salud, se les prescriba como modalidad terapéutica el uso de las plantas de Cannabis y sus derivados." Para poder llevar esto acabo, el artículo $8^{\circ}$ establece la creación de un registro nacional voluntario para aquellos pacientes que utilizan como tratamiento a sus patologías aceite de cáñamo y otros derivados de la planta de cannabis.

El acceso a los derivados de cannabis medicinal para aquellos con las patologías contempladas en el programa y que cuenten con la indicación médica pertinente se amplían: a) por un lado, según el artículo 7º, la Administración Nacional de Medicamentos, Alimentos y Tecnología Médica (ANMAT) permitirá la importación de aceite de cannabis y sus derivados; b) según el artículo $10^{\circ}$, el Estado nacional impulsará a través de los laboratorios de Producción Pública de Medicamentos nucleados en ANLAP, la producción pública de cannabis en todas sus variedades y su eventual industrialización en cantidades suficientes para su uso exclusivamente medicinal, terapéutico y de investigación.

Para ver un resumen de la situación en cada país, ver laTabla 1

Tabla 1. Comparación de los marcos normativos de los países seleccionados sobre el cannabis

\begin{tabular}{|c|c|c|c|c|}
\hline & ARGENTINA & URUGUAY & EEUU & HOLANDA \\
\hline Uso recreacional & Ilegal & Legal & $\begin{array}{l}\text { Ilegal en general excepto } \\
\text { en: } \\
\text { Alaska, California, Colora- } \\
\text { do, Maine, Massachusetts, } \\
\text { Nevada, Oregón, Vermont, } \\
\text { Washington, }\end{array}$ & Legal según (regiones) \\
\hline Uso medicinal & Legal & Legal & Legal en 30 estados & Legal \\
\hline Indicaciones & $\begin{array}{l}\text { Náuseas por quimiotera- } \\
\text { pia. Epilepsia refractaria. } \\
\text { Evidencia fuerte en: } \\
\text { Dolor crónico, espastici- } \\
\text { dad en esclerosis múl- } \\
\text { tiples, y la estimulación } \\
\text { de apetito en infecciones } \\
\text { por VIH. }\end{array}$ & $\begin{array}{l}\text { Epilepsia refractaria, } \\
\text { dolores crónicos y } \\
\text { neuropáticos }\end{array}$ & $\begin{array}{l}\text { Anorexia asociada a VIH. } \\
\text { Náuseas y vómitos - } \\
\text { quimioterapia. } \\
\text { Droga huérfana: } \\
\text { Síndrome de Dravet; } \\
\text { glioblastoma multiforme, } \\
\text { síndrome Lennox-Gastaut, } \\
\text { esquizofrenia pediátrica, y } \\
\text { glioma, espasmos infanti- } \\
\text { les, prevención de injerto } \\
\text { versus huésped, encefalo- } \\
\text { patía hipóxica isquémica } \\
\text { neonatal; síndrome del X } \\
\text { frágil. }\end{array}$ & $\begin{array}{l}\text { Espasticidad por esclerosis } \\
\text { múltiple }\end{array}$ \\
\hline $\begin{array}{l}\text { Producción y financia- } \\
\text { miento }\end{array}$ & $\begin{array}{l}\text { Pública (ANMAT importa } \\
\text { y provee gratuitamente a } \\
\text { los participantes del pro- } \\
\text { grama) }\end{array}$ & Pública & $\begin{array}{l}\text { Universidad de Mississip- } \\
\text { pi (para investigación) y } \\
\text { empresas privadas. }\end{array}$ & Pública \\
\hline Autocultivo & No regulado & Legal & $\begin{array}{l}\text { Dependiendo de la legisla- } \\
\text { ción de cada estado pue- } \\
\text { de ser ilegal, legal solo } \\
\text { para pacientes que la utili- } \\
\text { cen con fines medicinales, } \\
\text { o legal para uso recreacio- } \\
\text { nal. }\end{array}$ & $\begin{array}{l}\text { llegal (en debate parlamenta- } \\
\text { rio) }\end{array}$ \\
\hline
\end{tabular}

En un informe escrito de la ANMAT sobre los usos terapéuticos de los cannabinoides ${ }^{27}$, en los que se evaluaba su eficacia y seguridad, se analizaron distintas aplicaciones posibles, arribando a las siguientes conclusiones:

- Dolor crónico: existirían beneficios leves a moderados para el tratamiento del dolor, comparado a placebo. Pueden considerarse como opciones al asociarse a otras alternativas terapéuticas.

- Náuseas y vómitos debido a quimioterapia: los cannabinoides fueron 4 veces más efectivos que el placebo para el control de náuseas y vómitos.

- Estimulación del apetito en infecciones de HIV: la terapia con acetato megestrol demostró ser superior al uso de los cannabinoides.

- Espasticidad en esclerosis múltiples o paraplejia: los cannabinoides tendrían importancia en el caso de espasticidad no controlada con las terapéuticas habituales.

- Síndrome de Tourette: sin conclusiones.

- Epilepsia refractaria: reducción mayor o igual al $50 \%$ en la frecuencia de las convulsiones en el $47 \%$ de los pacientes tratados con CBD o su asociación con THC. Puede ser considerada como una alternativa adyuvante.

- Depresión, trastornos de ansiedad, trastornos del sueño, glaucoma, trastorno del humor, calidad de vida, ingesta calórica, aumento de peso: No existen evidencias sustentables $^{49}$. 
En el anexo 1 del reporte se mencionan las indicaciones del Marinol ${ }^{\circledR}$ (dronabinol) y Cesamet $^{\circledR}$ (nabilona) indicados para anorexia asociada con pérdida de peso en pacientes con SIDA (1992); náuseas y vómitos asociados con quimioterapia oncológica sin respuesta ante tratamientos antieméticos convencionales $(1985)^{49}$. Éstos junto al aceite de cannabis (permitido mediante importación por medio de autorización del RECANN) están aprobados por ANMAT. Por ende, podría concluirse que la ANMAT considera que hay evidencia científica suficiente para utilizar cannabinoides en caso de dolor crónico, espasticidad en esclerosis múltiple, y la estimulación de apetito en infecciones de HIV (en caso de no obtener resultados con el tratamiento convencional con megestrol). Además de las indicaciones referentes al tratamiento de patologías o sintomatologías específicas, también se considera el "uso compasivo" del cannabis en la disposición $840 / 1995^{53}$. Esto refiere a la autorización para administrar un producto farmacéutico antes que haya recibido su aprobación oficial en caso de enfermedades con alto riesgo de muerte o de severo deterioro de la calidad de vida en el caso de que no se encuentre disponible en el país un tratamiento eficaz ${ }^{54}$. Existen algunas situaciones en las cuales este uso estaría autorizado, por ejemplo: compromiso de vida (invalidez y discapacidad), falta de alternativas terapéuticas, autorización en otro país y pendiente de autorización local, etc. ${ }^{53}$. En estos casos las peticiones son evaluadas por un grupo de trabajo dentro del Departamento de Evaluación de Medicamentos y Afines, en colaboración o asesoramiento de profesionales, teniendo en cuenta además los reportes acerca de la eficacia y seguridad.

\section{Debates y actores sociales: desafíos}

Algunos de los desafíos pendientes incluyen aspectos relacionados con el autocultivo, la relación del uso medicinal con la regulación del uso recreacional; y el financiamiento e indicaciones medicinal del cannabis en patologías seleccionadas.

\section{Autocultivo}

En el marco legal actual, se identifican algunos actores claves, como la asociación "Mamá cultiva" que se dedican a la producción de aceite de cannabis manufacturado individualmente para tratar enfermedades como la epilepsia refractaria, que quedan fuera de la ley ${ }^{51}$. Según el artículo $5^{\circ}$ de la ley 23.737, para quienes no tuvieran una autorización, se establecen "condenas con prisión de cuatro a quince años y multas (...) a aquellos que cultive plantas (...) y produzca estupefacientes." Probándose que fuese para consumo personal, la pena sería reducida a un mes a dos años de prisión; pero el artículo 11ำ estipula que habría un aumento de la condena si la acción involucrarse menores ${ }^{50}$, como suele ocurrir en el uso del aceite de cannabis para las encefalopatías epilépticas en niños ${ }^{55}$.

El problema en esta cuestión reside en la dificultad y los costos que puede acarrear el conseguir los productos generados a partir del cannabis, siendo este el justificativo de las organizaciones pro-autocultivo. Otro actor relevante, es la ANMAT, que mostró su preocupación por la facilidad para la obtención del aceite ya que podría conducir a la elaboración de compuestos sin control que podrían derivar en muerte o discapacidad de mucha gente 27 .

\section{Financiamiento e indicaciones}

Otro conflicto que se puede vislumbrar a partir de la legislación vigente, y que se relaciona con el punto anterior, es el financiamiento y el acceso a los medicamentos. Sería necesario aclarar si estos deberían estar incluidos dentro del plan médico obligatorio (PMO) y si sus costos los debieran financiar, consecuentemente, el Estado, las obras sociales o prepagas. Un actor que se vería involucrado en esta cuestión es la futura Agencia Nacional de Evaluación de Tecnologías de Salud (AGNETS), quien deberá evaluar la efectividad, seguridad, la costo-efectividad de estos tratamientos y el impacto presupuestario que podrían generar las distintas opciones de financiación, para decidir cuál es la más conveniente.

Un factor a tener en cuenta es la capacitación del personal del sistema de salud sobre la administración del cannabis como tratamiento. Para esto sería necesario la generación de protocolos o guías de acción sobre cómo y en qué situaciones indicar esta alternativa terapéutica. La ANMAT ya ha realizado un informe sobre las posibles aplicaciones del cannabis, por lo que esta podría ser una de las herramientas a utilizar para informar al personal de salud, aunque es necesario mantener vigente la investigación y actualizar los datos que allí aparecen de manera periódica.

Otro factor que entra en juego en este aspecto de la financiación e indicaciones son los laboratorios y empresas farmacéuticas. Si bien la ley plantea que la ANMAT sea la encargada de proveer de manera gratuita los medicamentos mediante la importación de estos, y en un futuro por la producción nacional mediante la Agencia Nacional de Laboratorios Públicos (ANLAP) ${ }^{51}$, existen dudas acerca de qué posición tomarían los laboratorios privados al verse fuera de un negocio de tal magnitud.

Si se diera lugar a la producción por parte de los laboratorios privados una de las situaciones que podría darse sería la extensión de las aplicaciones del cannabis (uso off-label) para así abarcar a un mayor número de pacientes y de esa manera aumentar las ganancias para el sector (como sucede con otros fármacos) De esta forma sería obligación del Ministerio de Salud la regulación y control de las aplicaciones del cannabis.

\section{$R$ elación con uso recreacional}

También se debe tener en cuenta que el hecho del debate y legalización del uso de cannabis medicinal abre la puerta al debate sobre el consumo recreativo de la misma. Como fue mencionado anteriormente, la mayoría de los países que legislan de manera favorable sobre el uso medicinal del cannabis terminan haciendo lo mismo, o por lo menos flexibilizando sus legislaciones, en relación al consumo recreacional. A nivel institucional y social, en nuestro país este debate queda pendiente.

El hecho de que la legalización de la marihuana medicinal no esté acompañada por algún cambio en la legislación con respecto al consumo recreacional genera varias situaciones posibles. Por un lado, como fue mencionado anteriormente, la posibilidad de que si se autoriza el autocultivo medicinal podría devenir en el cultivo con fines recreacionales o actuar como incentivo para el comercio ilegal de cannabis. Otra situación para tener en cuenta sería la posibilidad de acceder al cannabis medicinal mediante recetas falsas con el fin de un uso recreacional. Esto podría darse mediante la falsificación de recetas por parte del usuario o por el comercio de estas por parte de profesionales de la salud que buscan un rédito económico personal. Por último, otro conflicto que surge entre el uso medicinal del cannabis y el uso recreacional es la estigmatización del cannabis. Podría llegar a ser preocupante que algunas personas, sobre todo pacientes de edad avanzada o grupos religiosos llegasen a rechazar un tratamiento eficaz para su padecimiento solo debido a que lo asocian con las consecuencias negativas, sociales y físicas que acarrea su uso desregulado o debido al temor del prejuicio de su grupo social. Dentro de los testimonios de "Mamá cultiva", se ha hecho una breve referencia al respecto, al mencionar como personas ajenas a la situación las acusan de drogar a sus hijos, aun cuando dichos casos buscan aliviar un padecimiento como la epilepsia refractaria y no un mero consumo recreacional ${ }^{55}$

\section{Potenciales soluciones}

- Producción nacional de cannabis y derivados

- Generación de "viveros comunitarios" 
- Registro electrónico de recetas de cannabis

- Financiamiento a partir del consumo recreacional

\section{Conclusiones}

De esta revisión se desprende como conclusión que son escasas las aplicaciones útiles del cannabis dentro del ámbito medicinal, pero que en aquellas que sí lo son, su utilización, permitiría mejorar las opciones de tratamiento del paciente. De esto se desprende la necesidad de una legislación integral, que no deje puntos relevantes por fuera de la ley. Consideramos que si bien en la Argentina ya existe una ley, la misma no es integral, por lo que se debería continuar el debate alrededor de la legislación y su implementación, para plantear los problemas y posibles soluciones que se desprenden del marco legal actual.

\section{Conflicto de interés de los autores: Ninguno}

Fuente de financiamiento: Ninguno

\section{Nota}

El presente texto constituye una versión abreviada del trabajo final integrador reflexivo de la asignatura Salud Pública de la carrera de medicina del Instituto Universitario Hospital Italiano.

\section{Referencias}

1. Atakan Z, et al. Cannabis, a complex plant: different compounds and different effects on individuals. Therapeutic Advances in Psychopharmacology. 2012;2(6):241-254

2. Wikimedia Foundation. Cannabidiol; 2018. Available from: https://es.wikipedia.org/wiki/Cannabidiol [Last access: 2019-05-22].

3. Wikimedia Foundation. Tetrahydrocannabinol; 2018. Available from: https://es.wikipedia.org/wiki/Tetrahidrocannabinol [Last access: 2018-05-07].

4. Osorio JH, Tangarife HF. Cannabis, una opción terapéutica. Biosalud. 2009;8(1):166-177. Available from: http://www.scielo.org.co/scielo.php?script= sci arttext\&pid=S1657-95502009000100019\&Ing=en\&nrm=iso.

5. Atance JR, Ruiz JF, Universidad Complutense. Uso de los cannabinoides a través de la historia. Adicciones, (SI) [En línea]. 2000;2(2):19-30. Available from: http://m.adicciones.es/index.php/adicciones/article/view/670/659.

6. Grotenhermen F, Russo E. Cannabis and cannabinoids: pharmacology, toxicology, and therapeutic potential. Routledge; 2008.

7. Yücel M, Lorenzetti V, et al. Hippocampal harms, protection and recovery following regular cannabis use. Translational Psychiatry. 2016;6(1).

8. Sneider J, Nickerson L, Silveri M. Cortical thinness and volume differences associated with marijuana abuse in emerging adults. Drug and Alcohol Dependence. Drug and Alcohol Dependence. 2015;155:275-83.

9. Solowij N, Jones KA, Rozman ME, Davis SM, Ciarrochi J, Heaven PC. Reflection impulsivity in adolescent cannabis users: a comparison with alcohol-using and non-substance-using adolescents. Psychopharmacology. 2011;2(575-86).

10. Nestoros JN, Vakonaki E, Tzatzarakis MN, Alegakis A, Skondras MD, Tsatsakis AM. Long lasting effects of chronic heavy cannabis abuse. The American Journal on Addictions. 2017;26(4):335-42.

11. Ogeil RP, Phillips JG, Rajaratnam SMW, Broadbear JH. Risky drug use and effects on sleep quality and daytime sleepiness. Human Psychopharmacology: Clinical and Experimental. 2017;5(356-63).

12. Hancox RJ, Shin H, Gray AR, Poulton R, Sears MR. Effects of quitting cannabis on respiratory symptoms. European Respiratory Journal. 2015;46(1):80-7.

13. Macleod J, Robertson R, Copeland L, Mckenzie J, Elton R, Reid P. Cannabis, tobacco smoking, and lung function: a cross-sectional observational study in a general practice population. British Journal of General Practice;2015(65).

14. Hackam DG. Cannabis and Stroke Systematic Appraisal of Case Reports. Stroke. 2015;46:852-856.

15. Salzwedel AP, Grewen K, Vachet C. Prenatal Drug Exposure Affects Neonatal Brain Functional Connectivity. Journal of Neuroscience. 2015;35(14):5860-9.

16. Varner M, RM S, Hogue C, Willinger M, Parker CB, Thorsten VR, et al. Association Between Stillbirth and Illicit Drug Use and Smoking During Pregnancy. Obstetrics \&amp. Gynecology. 2014;123(1):113-25.

17. Dines AM, Wood DM, Galicia M, Yates CM, Heyerdahl F, Hovda KE, et al. Presentations to the Emergency Department Following Cannabis use-a Multi-Centre Case Series from Ten European Countries. Journal of Medical Toxicology. 2015;11(4):415-21.

18. Herrmann E, Cone EJ, Mitchell JM, Bigelow GE, Lodico C, Flegel R, et al. Non-smoker exposure to secondhand cannabis smoke II: Effect of room ventilation on the physiological, subjective, and behavioral/cognitive effects. Drug and Alcohol Dependence. 2015;151:194-202.

19. Pardini D, White HR, Xiong S, Bechtold J, Chung T, Loeber R. Unfazed or Dazed and Confused: Does Early Adolescent Marijuana Use Cause Sustained Impairments in Attention and Academic Functioning? Journal of Abnormal Child Psychology. 2015;43(7):1203-17.

20. Greene MC, Kelly JF. The Prevalence of Cannabis Withdrawal and Its Influence on Adolescents' Treatment Response and Outcomes. Journal of Addiction Medicine. 2014;8(15):359-67.

21. Wang GS, Roosevelt G, Lait MC, Martintez EM, Bucher-Bartelson B, Bronstein A, et al. Association of Unintentional Pediatric Exposures With Decriminalization of Marijuana in the United States. Annals of Emergency Medicine. 2014;63(6):684-9.

22. Battistella G, Fornari E, Thomas A, Mall JF, Chtioui H, Appenzeller M, et al. Battistella G, Fornari E, Thomas A, Mall J-F, Chtioui H, Appenzeller M, et al. Weed or Wheel! fMRI, Behavioural, and Toxicological Investigations of How Cannabis Smoking Affects Skills Necessary for Driving. PLoS ONE. PLoS ONE. 2013;8(1):e52545.

23. Tateo S. State of the evidence: Cannabinoids and cancer pain-A systematic review. Journal of the American Association of Nurse Practitioners. 2017:29(2):94-103

24. Lynch ME, Ware MA. Cannabinoids for the Treatment of Chronic Non-Cancer Pain: An Updated Systematic Review of Randomized Controlled Trials. Journal of Neuroimmune Pharmacology. 2015;10(2):293-301.

25. Mücke M, Phillips T, Radbruch L, Petzke F, Häuser W. Cannabis-based medicines for chronic neuropathic pain in adults. Cochrane Database of Systematic Reviews. 2018;p. CD012182.

26. Stevens AJ, Higgins MD. A systematic review of the analgesic efficacy of cannabinoid medications in the management of acute pain. Acta Anaesthesiologica Scandinavica. 2017;61(3):268-80.

27. ANMAT. "Informe ultrarrápido de evaluación de tecnologías sanitaria. Uso terapeutico de los cannabinoides". Código interno: IURETS005 20160602 ANMAT[Fecha de acceso: 12/06/2018];: Available from: URL.

28. Wang T, Collet JP, Shapiro S, Ware MA. Adverse effects of medical cannabinoids: a systematic review. Canadian Medical Association Journal. 2008;178(13):1669-78.

29. Mücke M, Phillips T, Radbruch L, Petzke F, Häuser W. Systematic review and meta-analysis of cannabinoids in palliative medicine. Journal of Cachexia, Sarcopenia and Muscle. 2018;9(2):220-34.

30. MedlinePlus. Megestrol: MedlinePlus medicinas;. Available from: https://medlineplus.gov/spanish/druginfo/meds/a682003-es.html [Last access: 2018/07/05].

31. O'Connell BK, Gloss D, Devinsky O. Cannabinoids in treatment-resistant epilepsy: A review. Epilepsy \& Behavior. 2017;70:341-8.

32. Gross DW, Hamm J, Ashworth NL, Quigley D. Marijuana use and epilepsy: Prevalence in patients of a tertiary care epilepsy center. Neurology. 2004:62(11):2095-7. 
33. Lagae L, Brambilla I, Mingorance A, Gibson E, Battersby A. Quality of life and comorbidities associated with Dravet syndrome severity: a multinational cohort survey. Developmental Medicine \& Child Neurology. 2017;60(1):63-72.

34. O'Neil ME, Nugent SM, Morasco BJ, Freeman M, Low A, Kondo K, et al. Benefits and Harms of Plant-Based Cannabis for Posttraumatic Stress Disorder. Annals of Internal Medicine. 2017 sep;167(5):332. Available from: 10.7326/M17-0477.

35. Whiting PF, Wolff RF, Deshpande S, Nisio MD, Duffy S, Hernandez AV, et al. Cannabinoids for Medical Use. JAMA. 2015 jun;313(24):2456. Available from: 10.1001/jama.2015.6358.

36. Page SA, Verhoef MJ, Stebbins RA, Metz LM, Levy JC. Cannabis Use as Described by People with Multiple Sclerosis. Canadian Journal of Neurological Sciences / Journal Canadien des Sciences Neurologiques. 2003 aug;30(3):201-205. Available from: 10.1017/S0317167100002584.

37. Multiple Sclerosis;. Available from: https://www.safeaccessnow.org/ms [Last access: 2018/06/11]

38. Clark AJ, Ware MA. Yazer E, Murray TJ, Lynch ME. Patterns of cannabis use among patients with multiple sclerosis. Neurology. 2004;62(11):2098100 .

39. Kalmthout AV. Política holandesa en materia de drogas. Eguzkilore. 1988;(2):87-106. Available from: https://www.ehu.eus/documents/1736829/ 2162844/12+-+Aspectos+de+la+politica+holandesa.pdf.

40. Ferrer HMVE. Congreso holandés aprueba regular el cultivo legal de cannabis; 2017. Available from: https://elpais.com/internacional/2017/02/21/ actualidad/1487699905 727058.html.

41. Marihuana terapéutica en Holanda: el país que vende cannabis en las farmacias aún tiene mucho por hacer.; 2015. Available from: https://www. dinafem.org/es/blog/marihuana-terapeutica-holanda-farmacias/ [Last access: 2018/05/07].

42. Office of Medicinale Cannabis ; 2018. Available from: https://english.cannabisbureau.nl/ [Last access: 2018/05/07].

43. "Informe cannabinoides. Anexo 1. Situación ante las agencias de medicamentos en Países de alta Vigilancia Sanitaria";. Available from: http: //www.anmat.gov.ar/ets/Cannabinoides_Anexo_I.pdf [Last access: 2018/06/12].

44. Senado y la Cámara de Representantes de la República Oriental del Uruguay. Ley 19.172 [Uruguay]; 2014. Available from: https://legislativo. parlamento.gub.uy/temporales/leytemp585027.htm [Last access: 2018/05/07].

45. California Department of Public Health - Medical Marijuana Program, State of California. Medical Marijuana Program;. Available from: https://web.archive.org/web/20100101143455/http://www.cdph.ca.gov/programs/MMP/Pages/Medical\%20Marijuana\%20Program.aspx [Last access: 2019/05/29].

46. DEA[US]. Drug Schedulling;. Available from: https://www.dea.gov/drug-scheduling [Last access: 2019/05/29].

47. Joy JE, Watson SJ, Benson JA, Institute of Medicine (US). Marijuana and Medicine;Available from: https://medicalmarijuana.procon.org/sourcefiles/ IOM_Report.pdf.

48. Wikimedia Foundation. Medicamento huérfano;. Available from: https://es.wikipedia.org/wiki/Medicamento hu\%C3\%A9rfano [Last access: 2018/06/11].

49. ANMAT. "Informe cannabinoides. Anexo 1. Situación ante las agencias de medicamentos en Países de alta Vigilancia Sanitaria";. Available from: http://www.anmat.gov.ar/ets/Cannabinoides Anexo I.pdf [Last access: 2018/06/12].

50. Ley 27.735 - Codigo penal; 1989. Available from: http://servicios.infoleg.gob.ar/infoleglnternet/anexos/0-4999/138/texact.htm.

51. Ley 27.350 - Uso Medicinal de la Planta de Cannabis; 2017. Available from: http://www.saij.gob.ar/27350-nacional-ley-uso-medicinal-plantacannabis-Ins0006302-20 [Last access: 11/03/2018]

52. Reglamentación de Ley 27.350 - Uso Medicinal de la Planta de Cannabis;. Available from: http:/www.saij.gob.ar/738-nacional-reglamentacion-ley27350-sobre-investigacion-medica-cientifica-uso-medicinal-planta-cannabis-sus-derivados-dn20170000738-2017-09-21/123456789-0abc-8370000-7102soterced?q=\%28nl'\{u\}mero-norma\%3A738\%20AND\%20fecha\%3A2017\%29\%20 [Last access: 11/03/2018].

53. ANMAT. "Disposición 840/1995. Regulación de uso compasivo ”;. Available from: http://www.anmat.gov.ar/webanmat/Legislacion/Medicamentos/ Disposicion_840-1995.pdf [Last access: 12/06/2018].

54. ANMAT. "El acceso a medicamentos no registrados: LAs diferentes situaciones y sus particularidades";. Available from: http://www.anmat.gov.ar/ comunicados/Medicamentos_No_registrados_13-12-16.pdf [Last access: 12/06/2018].

55. Rodriguez-Alvarez J. Uso medicinal de la marihuana: Darle marihuana a un hijo; 2014. Available from: http://www.mamacultiva.org/wp-content/ uploads/2014/11/revista_viernes_nov2014.pdf. 